\title{
An Effective Approach of Shadow Detection and Removal using Edge Detection and Bilateral Filtering
}

\author{
Laxmi Singh Chandel \\ Department of Computer Science \& Engineering \\ Samrat Ashok Technological Institute \\ Vidisha, India
}

\author{
Ajay Kumar Goyal \\ Department of Computer Science \& Engineering \\ Samrat Ashok Technological Institute \\ Vidisha, India
}

\begin{abstract}
Here in this paper automatic Shadow detection with Shadow Removal technique is implemented. The planned procedure implemented is based on the concept of identifying the Shadow region for the recognition of Shadow and then applying Filtering over the region to eliminate the silhouette consequence from the image. The planned procedure implemented here works in Two Stages, in the first stage Hybrid technique of arbitrary Walker Segmentation with Canny Edge Detector and bilateral filtering is applied in next Stage. The planned method implemented here is applied over dataset and the scheme provide better Positive rate and High True Positive rate and low error rate.
\end{abstract}

\section{Keywords}

Shadow Detection, Shadow Removal, Canny Edge Detection, Bilateral Filtering, Random Walker Segmentation.

\section{INTRODUCTION}

A shadow which is recognized to be cause by the interaction of light with objects claims a fraction of the image surface. Shadows in images hold importance for a variety of reasons. On one hand, shadows may lead to the failure of image analysis processes and also cause deterioration in the quality of information which in turn leads to problems procedures in completion of articulate for prospect considerate, entity gratitude and numerous additional applications. On the additional offer, they aid in sequence as cues in construction uncovering, alleyway verdict etc. [1], because, darkness occasionally produce objectionable belongings on descriptions, shadow modernization is measured imperative, and recognition of darkness is the foremost tread towards the purpose. In addition, to belongings somewhere they be active as an minister to, darkness recognition in descriptions the stage a crucial responsibility. It is second-hand for terra firma monitoring, distant sensing, modify uncovering, representation segmentation, countenance gratitude etc.

\subsection{Overview of Shadow}

\section{A. Shadow}

A silhouette takes place when an entity incompletely or completely occurs straight brightness from a foundation of enlightenment. If the brightness liveliness is fallen a smaller amount, that neighborhood is represent as silhouette district while if the brightness power is emit additional this neighborhood is represent as non silhouette district.

\section{B. Identity and Shed Shadow}

Shade can be confidential into two categories: cast shadow and personality silhouette. Direct silhouette is provided by the protuberance of the brightness foundation in the course of the object. Self outline is unmoving a silhouette but represent the ingredient of the article that is not illuminated unswervingly by the brightness source. Another time throw silhouette can be confidential into two parts: umbra and partial shade. The division of a throw silhouette wherever through brightness is entirely infertile by its objective is called umbra, whereas the ingredient where unswerving brightness is incompletely infertile is called obscurity [2].

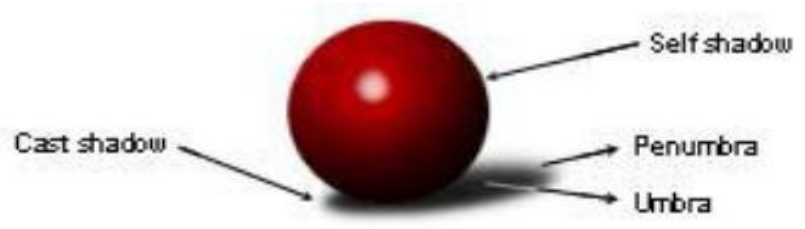

Fig.1 Diverse types of outline

\subsection{Various Shadow Detection Methods}

As described in [4], silhouette uncovering technique can be categorized as: model based and property based as proposed.

\begin{abstract}
A. Replica Based Procedure
Replica based procedures have incomplete applicability and are functional to precise troubles (say mid-air images) and simple objects only. The reliant on proceeding in sequence about lighting circumstances and prospect geometry as glowing as the entity which also turns out to be a chief disadvantage [2]. These procedures rotate approximately the thought that the desired structures do have repetitive geometries. Hence, probabilistic models could be easily implemented for segmentation purpose. Also, various sets of geometric features are matched with three dimensional object models [4].
\end{abstract}

\section{B. Property Based Techniques}

Possessions based silhouette uncovering methods have smaller amount confines as compared to the mock-up based procedures. They are recognized to be a immense deal improved, because, in these not only geometrical facial appearance are used but they are also combined with the spectral properties of shadow like color or brightness etc. These tactics have been widely used in literature since these are straightforward and trouble-free to execute. As affirmed in [5], these can be generally confidential as: i) thresholding based; ii) shade transformation based; iii) district mounting based; and iv) classification-based. Reference [6] summarizes the categorization of silhouette uncovering procedures specified in [7] anywhere affecting direct silhouette uncovering method have been confidential as: (i) shade/gamut based procedure; (ii) surface based procedure, and (iii) geometry based procedures. According to [8] moving shadows can be detected based on: (i) intensity information, (ii) photometric changing in sequence and (iii) shade and arithmetical in sequence. Also, in [10] shadow removal methods have been categorized as: (i) chromaticity-based 
methods; (ii) physical methods; (iii) geometrical-based methods and (iv) texture-based methods.

\section{2. LITERATURE SURVEY}

Song et al. [5], Luus et al. [9], Kuo et al. [10] proposed a new Threshold based scheme for the Shadow Detection. Predefined entrance height based on bimodal histogram used to conclude silhouette and non outline pixels. The technique implemented is simple and fast. Requires post dispensation as consequences strength be disjointed or indistinct and possibly will contain holes, sound etc.

Golchin et al. [11], Leone et al. [12], Leone et al. [13], Heikkila et al. [14] proposed another silhouette uncovering technique based on the concept of Texture based Shadow Detection. On this report the correspondence stuck between environment and darkness consistency as fighting fit as the divergence in forefront and surroundings textures. The Technique Accurate results under stable illumination conditions. Best for indoor scenes. Difficult to implement. Poor performance for outdoor scenes as texture cannot be captured.

$\mathrm{Xu}$ et al. [15], Xu et al. [16] proposed a new scheme which is based on Region Growing based technique. kernel point are chosen. Those groups of pixels with elevated self-confidence of organism silhouette (say according to detachment) and silhouette neighborhood complete, enlargement forbidden by connectivity. It will appropriately disconnect silhouette and non silhouette regions. Edges are crisp.

Guo et al. [17], Liu et al.[18] also proposed a new scheme based on Classification methods. Classification procedures like SVM are used based on the concepts overcome by silhouette pixels. It will distinguish plausible gloominess limitations precisely. Straightforward and effortless to put into practice. There are chances of misclassification. Shadows of small objects are missed sometimes.

Golchin et al. [11], Asaidi et al. [19] proposed Geometric Properties based Shadow Detection technique. Sets of geometric features are matched. Effective detection under simulated and controlled environment. Huge calculation. Not practicable for spatial, genuine occasion cases. reliant on entity prospect connection.

\section{PROPOSED METHODOLOGY}

Here for the Automatic silhouette uncovering and taking away from Images Hybrid Technique of applying Random Walker Segmentation with Canny Edge Detector and bilateral filtering is used.

\subsection{Random Walker Segmentation}

It is a method of segmentation on the foundation of selecting foreground and background as seed pixels by moving randomly to other pixels moving from background till any foreground pixel is obtained and the region is extracted as segmented region from the representation.

\subsection{Canny Edge Detector}

Crafty edge uncovering technique finds boundaries by looking for restricted maxima of the gradiant of $f(x, y)$. Here the incline charge is calculating by means of the plagiaristic of a Gaussian Filter. The move toward second-hand here will take two threshold to find brawny and feeble boundaries, and contain the weak edges in the output only if they are connected to strong edges. Therefore, this approach is additional likely to detect true weak edges.
1. Allocate region seeds si for each region I

2. Calculate ui $(x, y)$ : the likelihood of original inward si for a haphazard rambler opening from $(\mathrm{x}, \mathrm{y})$

3. Dispense $(\mathrm{x}, \mathrm{y})$ to marker $\mathrm{k}$ if $\mathrm{uk}(\mathrm{x}, \mathrm{y})$ is the principal amongst ui $(\mathrm{x}, \mathrm{y})$ for $\mathrm{i}=1 \ldots \mathrm{N}$

\section{Steps Involved in Canny Method}

- The image is smoothed using Gaussian strain with a particular paradigm departure, $\sigma$, to diminish noise

- The confined incline indicate $\mathrm{g}(\mathrm{x}, \mathrm{y})$ and periphery bearing are compute at each indicate.

- The boundary indicate unwavering give ascend to ridges in the pitch enormity image. This crumple pixels are then thresholds, $\mathrm{T} 1 \& \mathrm{~T} 2$, with $\mathrm{T} 1<\mathrm{T} 2$.

\subsection{Bilateral Filtering}

A bilateral filter is a non-linear, edge-preserving, and noisereducing smoothing filter for images. This heaviness can be categorize on a Gaussian allocation.

The two-pronged pass through a filter is definite as

$$
I^{\text {filtered }}(x)=\frac{1}{W_{p}} \sum_{x_{i} \in \Omega} I\left(x_{i}\right) f_{r}\left(\left\|I\left(x_{i}\right)-I(x)\right\|\right) g_{s}\left(\left\|x_{i}-x\right\|\right),
$$

where the normalization term

$$
W_{p}=\sum_{x_{i} \in \Omega} f_{r}\left(\left\|I\left(x_{i}\right)-I(x)\right\|\right) g_{s}\left(\left\|x_{i}-x\right\|\right)
$$

ensures that the sieve preserves representation energy and

- $I^{\text {filtered }}$ is the filtered image;

- $I$ is the unusual participation likeness to be drinkable;

- $x$ are the coordinate of the present pixel to be clean;

- $\Omega$ is the casement centered in $x$;

- $f_{r}$ is the assortment kernel for filtered variation in intensities. This purpose can be a Gaussian purpose;

- $g_{s}$ is the spatial essential part for smoothing variation in coordinates. This purpose can be a Gaussian meaning;

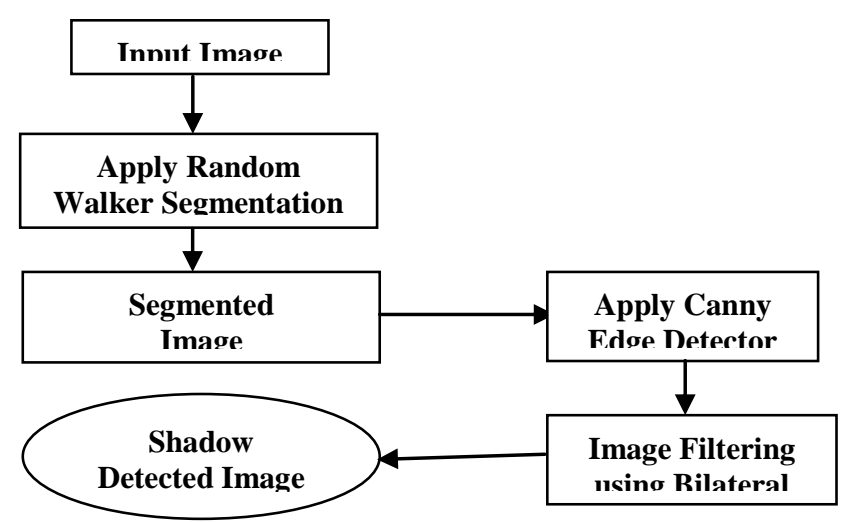

Fig. 2 Flow Chart of Proposed Methodology 


\section{RESULT ANALYSIS}

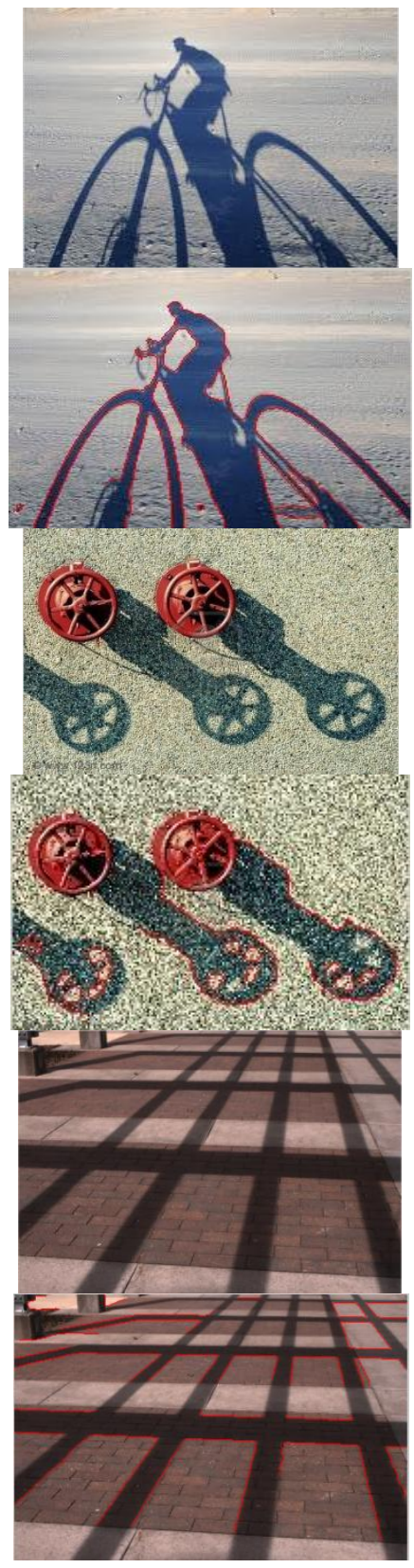

Fig. 3 Shadow Detection using Proposed Framework

The table shown below is the analysis and comparison of Existing and proposed schemes evaluated on the source of their routine of Shadow Detection. The planned Procedure implemented here for various Datasets provides high Performance of Shadow Detection.

Table 1. Evaluation of the proposed Shadow Detection Scheme

\begin{tabular}{|c|c|c|c|}
\hline Methods & $\begin{array}{c}\text { UCF } \\
\text { Dataset }\end{array}$ & $\begin{array}{c}\text { CMU } \\
\text { Dataset }\end{array}$ & $\begin{array}{c}\text { UIUC } \\
\text { Dataset }\end{array}$ \\
\hline Existing Work & 90.65 & 88.79 & 93.16 \\
\hline Proposed Work & 92.63 & 91.27 & 94.83 \\
\hline
\end{tabular}

The table shown below is the analysis and comparison of Existing and proposed schemes evaluated on the basis of their Root Mean Square Error of Shadow Detection. The planned method procedure here for various Datasets provides Less RMSE of Shadow Detection.

Table 2. Quantitative Analysis of RMSE per Pixel

\begin{tabular}{|c|c|c|c|}
\hline Methods & $\begin{array}{c}\text { Shadow } \\
\text { Region }\end{array}$ & $\begin{array}{c}\text { Literal } \\
\text { Region }\end{array}$ & All Region \\
\hline Existing Work & 10.5 & 4.7 & 6.1 \\
\hline Proposed Work & 8.64 & 3.2 & 5.23 \\
\hline
\end{tabular}

The Figure shown below is the analysis and comparison of Existing and proposed schemes evaluated on the foundation of their presentation of Shadow Detection. The planned method implemented here for various Datasets provides high Performance of Shadow Detection.

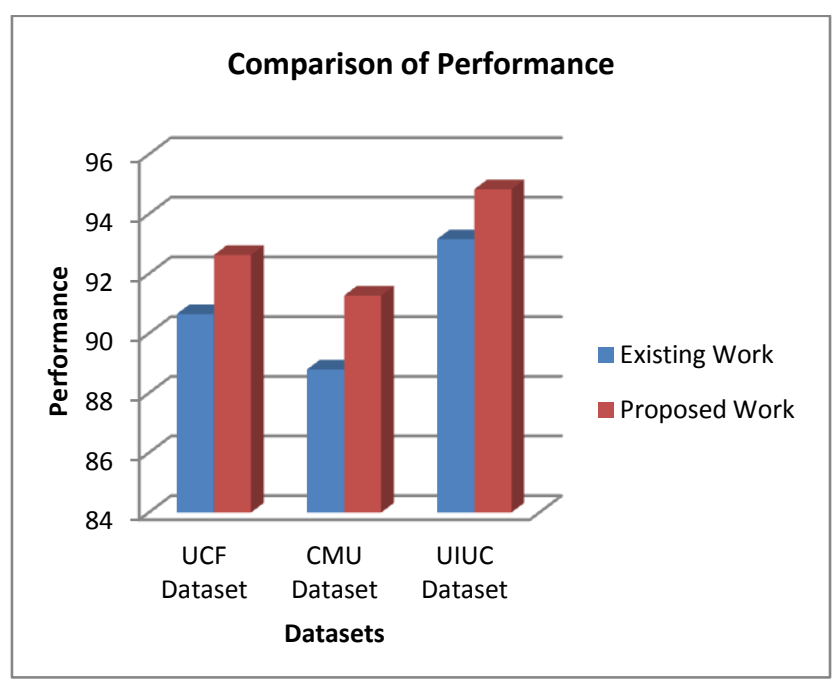

Fig. 4 Comparison of Performance Evaluation

The Figure shown below is the analysis and comparison of Existing and proposed schemes evaluated on the basis of their Root Mean Square Error of Shadow Detection. The planned tactic implemented here for various Datasets provides Less RMSE of Shadow Detection.

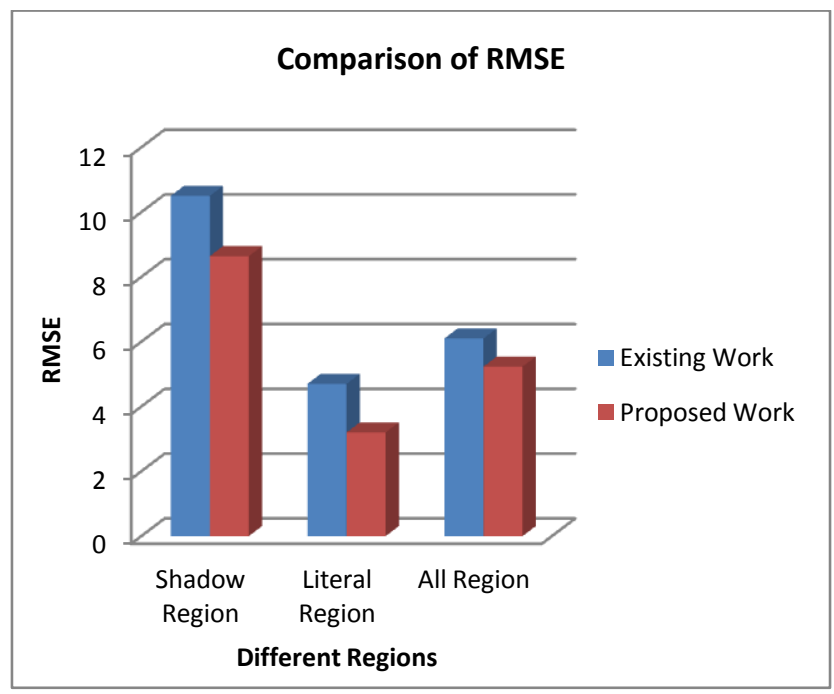

Fig. 5 Comparison of RMSE per pixel 


\section{CONCLUSION}

Shadow Detection is the identification of Shadow from the images and applying some filtering technique over the shadows to filter the effect of Shadows from the images. Here a new and efficient technique for the uncovering of silhouette using Canny Edge Detection and two-pronged filtering is used for the Shadow uncovering and Removal. The Proposed Scheme is compared with the existing silhouette uncovering and elimination scheme and finds that the scheme implemented here provides High Positive rate and better Accuracy of silhouette uncovering and Low Error rate.

\section{REFERENCES}

[1] Arevalo V, González J, Ambrosio G, "Detecting Shadow QuickBird satellite images," ISPRS 2006 Commission VII Mid-term Symposium 'Remote Sensing: From Pixels to Processes'. Enschede, the Netherlands, 8-11 May, pp. 330,335

[2] Rajni Thakur, Shveta Chadda, Navjeet Kaur: "Review on Shadow Detection and Removal Techniques/Algorithms", International Journal of Computer Science And Technology, ISSN: 0976-8491, Volume 3, Issue 1, Jan - March 2012.

[3] Shwetali Wakchaure, Poorva Raut: "A Review of Shadow Detection and Reconstruction in VHR Images", The International Journal Of Science and Technoledge (IJST), ISSN: 2321-919X, Volume 2, Issue 3, March2014.

[4] E. Salvador, A. Cavallaro, and T. Ebrahimi, "Cast shadow segmentation using invariant color features," Comput. Vis. Image Understand., vol. 95, no. 2, pp. 238-259, Aug. 2004. roc. Computer Vision and Image Understanding 95(2004) 238-259.

[5] Huihui Song; Bo Huang; Kaihua Zhang, "Shadow Detection and Reconstruction in High-Resolution Satellite Images via Morphological Filtering and Example-Based Learning," IEEE Transactions on Geoscience and Remote Sensing, , vol.52, no.5, pp.2545,2554, May 2014.

[6] Ariel Amato, Ivan Huerta, Mikhail G. Mozerov, F. Xavier Roca and Jordi Gonz`alez: "Moving Cast shadow Detection Methods for Video surveillance Application," pp. 1-25 (2013).

[7] Wei Zhang, Q.M. Jonathan Wu, and Xiangzhong Fang, "Vision Systems: Segmentation and Pattern Recognition. Moving Cast Shadow Detection," Goro Obinata and Ashish Dutta, InTech

[8] Habib Ullah, Mohib Ullah, Muhammad Uzair, and Fasih ur Rehman, "Comparative study: The evaluation of shadow detection methods," International Journal Of Video \& Image Processing And Network Security (IJVIPNS), Vol.10(2), pp.1,7, April 2010.
[9] Luus, F.P.S.; van den Bergh, F.; Maharaj, B.T.J., "Adaptive Threshold-Based Shadow Masking for Across-Date Settlement Classification of Panchromatic QuickBird Images," Geoscience and Remote Sensing Letters, IEEE, vol.11, no.6, pp.1153,1157, June 2014

[10] Kuo-Liang Chung; Yi-Ru Lin; Yong-Huai Huang, "Efficient Shadow Detection of Color Aerial Images Based on Successive Thresholding Scheme," , IEEE Transactions on Geoscience and Remote Sensing, vol.47, no.2, pp.671,682, Feb. 2009

[11] Maryam Golchin, Fatimah Khalid, Lili Nurliana Abdullah and Seyed Hashem Davarpanah, 'Shadow Detection Using Color and Edge Information', Journal of Computer Science, Vol. 9 (11), pp.1575,1588, 2013.

[12] Leone, A.; Distante, C.; Buccolieri, F., "A texture-based approach for shadow detection," IEEE Conference on Advanced Video and Signal Based Surveillance, 2005. AVSS 2005., vol., no., pp.371,376, 15-16 Sept. 2005

[13] A.Leone, C.Distante, "Shadow Detection for Moving Objects based on Texture analysis," Pattern Recognition, Vol. 40(4), pp. 1222,1233, April 2007

[14] Heikkila, M.; Pietikainen, M., "A texture-based method for modeling the background and detecting moving objects," IEEE Transactions on Pattern Analysis and Machine Intelligence,, vol.28, no.4, pp.657,662, April 2006.

[15] Dong Xu; Jianzhuang Liu; Zhengkai Liu; Xiaoou Tang, "Indoor shadow detection for video segmentation," IEEE International Conference on Multimedia and Expo, 2004. ICME '04. 2004, vol.1, no., pp.41,44 Vol.1, 27-30 June 2004.

[16] Dong Xu; Jianzhuang Liu; Xuelong Li; Zhengkai Liu; Xiaoou Tang, "Insignificant shadow detection for video segmentation," IEEE Transactions on Circuits and Systems for Video Technology , vol.15, no.8, pp.1058,1064, Aug. 2005.

[17] Ruiqi Guo; Qieyun Dai; Hoiem, D., "Single-image shadow detection and removal using paired regions," IEEE Conference on Computer Vision and Pattern Recognition (CVPR), 2011, vol., no., pp.2033,2040, 2025 June 2011

[18] Xin Liu; Bin Dai; Hangen He, "Real-Time On-Road Vehicle Detection Combining Specific Shadow Segmentation and SVM Classification," II Second International Conference on Digital Manufacturing and Automation (ICDMA),IEEE,2011, vol., no., pp.885,888, 5-7 Aug. 2011

[19] Asaidi, H.; Aarab, A.; Bellouki, M., "Shadow detection approach combining spectral and geometrical properties," IEEE International Conference on Multimedia Computing and Systems (ICMCS), 2012, vol., no., pp.389-393, 10-12 May 2012 